KYUNGPOOK Math. J. 54(2014), 453-461

http://dx.doi.org/10.5666/KMJ.2014.54.3.453

\title{
Certain Class of Analytic Functions Defined by Ruscheweyh Derivative with Varying Arguments
}

\author{
RaBha Mohamed EL-Ashwah \\ Department of Mathematics, Faculty of Science, Damietta University, New Dami- \\ etta 34517, Egypt \\ e-mail : r_elashwah@yahoo.com \\ Mohamed Kamal Aouf \\ Department of Mathematics, Faculty of Science, Mansoura University, Mansoura \\ 33516, Egypt \\ e-mail : mkaouf127@yahoo.com \\ Ahmed Hassan And AlaA Hassan* \\ Department of Mathematics, Faculty of Science, Zagazig University, Zagazig 44519, \\ Egypt \\ e-mail : aam_hassan@yahoo.com, alaahassan1986@yahoo.com
}

ABstract. In this paper we derive some results for certain new class of analytic functions defined by using Ruscheweyh derivative with varying arguments.

\section{Introduction}

Let $\mathcal{A}$ denote the class of functions of the form:

$$
f(z)=z+\sum_{k=2}^{\infty} a_{k} z^{k}
$$

which are analytic and univalent in the open unit disc $U=\{z \in \mathbb{C}:|z|<1\}$. Given

* Corresponding Author.

Received September 17, 2012; accepted April 22, 2013.

2010 Mathematics Subject Classification: 30C45.

Keywords and Phrases: analytic functions, univalent, Hadamard product, Ruscheweyh derivative, extreme points. 
two functions $f, g \in \mathcal{A}$, where $f(z)$ is given by (1.1) and $g(z)$ is given by

$$
g(z)=z+\sum_{k=2}^{\infty} b_{k} z^{k}
$$

the Hadamard product $(f * g)(z)$ is defined by

$$
(f * g)(z)=z+\sum_{k=2}^{\infty} a_{k} b_{k} z^{k}=(g * f)(z) .
$$

By using the Hadamard product, Ruscheweyh [7] defined

$$
D^{\gamma} f(z)=\frac{z}{(1-z)^{\gamma+1}} * f(z) \quad(\gamma \geq-1) .
$$

Also Ruscheweyh [7] observed that

$$
D^{n} f(z)=\frac{z\left(z^{n-1} f(z)\right)^{(n)}}{n !}\left(n \in \mathbb{N}_{0}=\mathbb{N} \cup\{0\} ; \mathbb{N}=\{1,2, \ldots\}\right) .
$$

The symbol $D^{n} f(z)\left(n \in \mathbb{N}_{0}\right)$ was called the $n$-th order Ruscheweyh derivative of $f(z)$ by Al-Amiri [1]. We note that

$$
D^{0} f(z)=f(z) \text { and } D^{1} f(z)=z f^{\prime}(z) .
$$

It is easy to see that

$$
D^{n} f(z)=z+\sum_{k=2}^{\infty} \delta(n, k) a_{k} z^{k}
$$

where

$$
\delta(n, k)=\left(\begin{array}{c}
n+k-1 \\
n
\end{array}\right) .
$$

In [4] Attiya and Aouf defined the class $Q(n, \lambda, A, B)$ as follows:

Definition 1.([4]) Let $Q(n, \lambda, A, B)$ denote the subclass of $\mathcal{A}$ consisting of functions $f(z)$ of the form (1.1) such that

$$
\begin{gathered}
(1-\lambda)\left(D^{n} f(z)\right)^{\prime}+\lambda\left(D^{n+1} f(z)\right)^{\prime} \prec \frac{1+A z}{1+B z} \\
\left(\lambda \geq 0 ;-1 \leq A<B \leq 1 ; 0<B \leq 1 ; n \in \mathbb{N}_{0} ; z \in U\right) .
\end{gathered}
$$

Specializing the parameters $\lambda, A, B$ and $n$, we can obtained different classes studied by various authors: 
(i) $Q(0, \lambda, 2 \alpha-1,1)=R(\lambda, \alpha)(0 \leq \alpha<1, \lambda \geq 0)$ (see Altintas [3])

(ii) $Q(0,0,2 \alpha-1,1)=T^{* *}(\alpha)(0 \leq \alpha<1)$ (see Sarangi and Uralegaddi [8] and Al-Amiri [2])

(iii) $Q(n, 0,2 \alpha-1,1)=Q_{n}(\alpha)\left(0 \leq \alpha<1, n \in \mathbb{N}_{0}\right)$ (see Uralegaddi and Sarangi $[11])$

(iv) $Q(0,0,(2 \alpha-1) \beta, \beta)=P^{*}(\alpha, \beta)(0 \leq \alpha<1,0<\beta \leq 1)$ (see Gupta and Jain $[5])$

(v) $Q(0,0,((1+\mu) \alpha-1) \beta, \mu \beta)=P^{*}(\alpha, \beta, \mu)(0 \leq \alpha<1,0<\beta \leq 1,0<\mu \leq 1)$ (see Owa and Aouf $[6]$ ).

Also we note that:

(i) $Q(0, \lambda, A, B)=R(\lambda, A, B)$

$$
=\left\{f(z) \in \mathcal{A}: f^{\prime}(z)+\lambda z f^{\prime \prime}(z) \prec \frac{1+A z}{1+B z} \quad(\lambda \geq 0 ;-1 \leq A<B \leq 1 ; 0<B \leq 1 ; z \in U)\right\}
$$

(ii) $Q(n, 0, A, B)=Q_{n}(A, B)=$

$$
\left\{f(z) \in \mathcal{A}:\left(D^{n} f(z)\right)^{\prime} \prec \frac{1+A z}{1+B z} \quad\left(\lambda \geq 0 ;-1 \leq A<B \leq 1 ; 0<B \leq 1 ; n \in \mathbb{N}_{0} ; z \in U\right)\right\}
$$

Silverman [9] defined the class of univalent functions $f(z)=z+\sum_{k=2}^{\infty} a_{k} z^{k}$ for which $\arg \left(a_{k}\right)$ prescribed in such a way that $f(z)$ is univalent if and only if $f(z)$ is starlike as follows:

Definition 2. ([9]) A function $f(z)$ of the form (1.1) is said to be in the class $V\left(\theta_{k}\right)$ if $f \in \mathcal{A}$ and $\arg \left(a_{k}\right)=\theta_{k}$ for all $k \geq 2$. If further more there exist a real number $\delta$ such that $\theta_{k}+(k-1) \delta \equiv \pi(\bmod 2 \pi)(k \geq 2)$, then $f(z)$ is said to be in the class $V\left(\theta_{k}, \delta\right)$. The union of $V\left(\theta_{k}, \delta\right)$ taken over all possible sequences $\left\{\theta_{k}\right\}$ and all possible real numbers $\delta$ is denoted by $V$.

Let $V Q(n, \lambda, A, B)$ denote the subclass of $V$ consisting of functions $f(z) \in$ $Q(n, \lambda, A, B)$.

We note that $V Q(0,0,2 \alpha-1,1)=C_{\alpha}(0 \leq \alpha<1)=\left\{f \in V: \operatorname{Re}\left\{f^{\prime}(z)\right\}>\alpha\right\}$, studied by Srivastava and Owa [10].

Also we note that by specializing the parameters $\lambda, A, B$ and $n$ we can obtain different classes with varying arguments:

(i) $\operatorname{VQ}(0, \lambda, 2 \alpha-1,1)=\operatorname{VR}(\lambda, \alpha)(0 \leq \alpha<1, \lambda \geq 0)$

(ii) $V Q(0,0,2 \alpha-1,1)=V T^{* *}(\alpha)(0 \leq \alpha<1)$

(iii) $V Q(n, 0,2 \alpha-1,1)=V Q_{n}(\alpha)\left(0 \leq \alpha<1, n \in \mathbb{N}_{0}\right)$ 
(iv) $V Q(0,0,(2 \alpha-1) \beta, \beta)=V P^{*}(\alpha, \beta)(0 \leq \alpha<1,0<\beta \leq 1)$

(v) $V Q(0,0,((1+\mu) \alpha-1) \beta, \mu \beta)=V P^{*}(\alpha, \beta, \mu)(0 \leq \alpha<1,0<\beta \leq 1,0<$ $\mu \leq 1)$

(vi) $V Q(0, \lambda, A, B)=V R(\lambda, A, B)(\lambda \geq 0,-1 \leq A<B \leq 1,0<B \leq 1)$

(vii) $V Q(n, 0, A, B)=V Q_{n}(A, B)\left(-1 \leq A<B \leq 1,0<B \leq 1, n \in \mathbb{N}_{0}\right)$

In this paper we obtain coefficient bounds for functions in the class $V Q(n, \lambda, A, B)$, further we obtain distortion bounds and the extreme points for functions in this class.

\section{Coefficient Estimates}

Unless otherwise mentioned, we assume in the reminder of this paper that, $\lambda \geq 0,-1 \leq A<B \leq 1,0<B \leq 1, n \in \mathbb{N}_{0}, \delta(n, k)$ and $C_{k}$ are given by (1.7) and (2.2) respectively and $z \in U$.

Theorem 1. Let the function $f(z)$ defined by (1.1) be in $V$. Then $f(z) \in$ $V Q(n, \lambda, A, B)$, if and only if

$$
\sum_{k=2}^{\infty} k \delta(n, k) C_{k}\left|a_{k}\right| \leq(B-A)(n+1),
$$

where

$$
C_{k}=(1+B)[n+1+\lambda(k-1)] .
$$

Proof. Suppose that $f(z) \in V Q(n, \lambda, A, B)$. Then

$$
h(z)=(1-\lambda)\left(D^{n} f(z)\right)^{\prime}+\lambda\left(D^{n+1} f(z)\right)^{\prime}=\frac{1+A w(z)}{1+B w(z)},
$$

where

$$
w \in H=\{w \text { analytic, } w(0)=0 \text { and }|w(z)|<1, z \in U\} .
$$

Thus we get

$$
w(z)=\frac{1-h(z)}{B h(z)-A} .
$$

Therefore

$$
h(z)=1+\sum_{k=2}^{\infty} \frac{k[n+1+\lambda(k-1)] \delta(n, k)}{n+1} a_{k} z^{k-1},
$$

and $|w(z)|<1$ implies

$$
\left|\frac{\sum_{k=2}^{\infty} \frac{k[n+1+\lambda(k-1)] \delta(n, k)}{n+1} a_{k} z^{k-1}}{(B-A)+B \sum_{k=2}^{\infty} \frac{k[n+1+\lambda(k-1)] \delta(n, k)}{n+1} a_{k} z^{k-1}}\right|<1 .
$$


Since $f(z) \in V, f(z)$ lies in the class $V\left(\theta_{k}, \delta\right)$ for some sequence $\left\{\theta_{k}\right\}$ and a real number $\delta$ such that

$$
\theta_{k}+(k-1) \delta \equiv \pi(\bmod 2 \pi) \quad(k \geq 2) .
$$

Set $z=r e^{i \delta}$ in $(2.4)$, we get

$$
\left|\frac{\sum_{k=2}^{\infty} \frac{k[n+1+\lambda(k-1)] \delta(n, k)}{n+1}\left|a_{k}\right| r^{k-1}}{(B-A)-B \sum_{k=2}^{\infty} \frac{k[n+1+\lambda(k-1)] \delta(n, k)}{n+1}\left|a_{k}\right| r^{k-1}}\right|<1 .
$$

Since $\operatorname{Re}\{w(z)\}<|w(z)|<1$, we have

$$
\operatorname{Re}\left\{\frac{\sum_{k=2}^{\infty} \frac{k[n+1+\lambda(k-1)] \delta(n, k)}{n+1}\left|a_{k}\right| r^{k-1}}{(B-A)-B \sum_{k=2}^{\infty} \frac{k[n+1+\lambda(k-1)] \delta(n, k)}{n+1}\left|a_{k}\right| r^{k-1}}\right\}<1 .
$$

Hence

$$
\sum_{k=2}^{\infty} k C_{k} \delta(n, k)\left|a_{k}\right| r^{k-1} \leq(B-A)(n+1) .
$$

Letting $r \longrightarrow 1$ in (2.7), we get (2.1).

Conversely, $f(z) \in V$ and satisfies (2.1). Since $r^{k-1}<1$. So we have

$$
\begin{aligned}
\mid \sum_{k=2}^{\infty} \frac{k[n+1+\lambda(k-1)] \delta(n, k)}{n+1} & \left|a_{k}\right| z^{k-1}\left|\leq \sum_{k=2}^{\infty} \frac{k[n+1+\lambda(k-1)] \delta(n, k)}{n+1}\right| a_{k} \mid r^{k-1} \\
\leq & (B-A)-B \sum_{k=2}^{\infty} \frac{k[n+1+\lambda(k-1)] \delta(n, k)}{n+1}\left|a_{k}\right| r^{k-1} \\
\leq & (B-A)-B \sum_{k=2}^{\infty} \frac{k[n+1+\lambda(k-1)] \delta(n, k)}{n+1} a_{k} z^{k-1} \mid \\
& \leq\left|(B-A)+B \sum_{k=2}^{\infty} \frac{k[n+1+\lambda(k-1)] \delta(n, k)}{n+1} a_{k} z^{k-1}\right|
\end{aligned}
$$

which gives (2.4) and hence follows that

$$
(1-\lambda)\left(D^{n} f(z)\right)^{\prime}+\lambda\left(D^{n+1} f(z)\right)^{\prime}=\frac{1+A w(z)}{1+B w(z)}
$$

that is $f(z) \in V Q(n, \lambda, A, B)$. This completes the proof of Theorem 1 . 
Corollary 1. Let the function $f(z)$ defined by (1.1) be in the class $V Q(n, \lambda, A, B)$. Then

$$
\left|a_{k}\right| \leq \frac{(B-A)(n+1)}{k C_{k} \delta(n, k)}(k \geq 2) .
$$

The result (2.1) is sharp for the function $f(z)$ defined by

$$
f(z)=z+\frac{(B-A)(n+1)}{k C_{k} \delta(n, k)} e^{i \theta_{k}} z^{k}(k \geq 2) .
$$

\section{Distortion Theorems}

Theorem 2. Let the function $f(z)$ defined by (1.1) be in the class $V Q(n, \lambda, A, B)$. Then

$$
|z|-\frac{B-A}{C_{2}}|z|^{2} \leq|f(z)| \leq|z|+\frac{B-A}{C_{2}}|z|^{2} .
$$

The result is sharp.

Proof. We employ the same technique as used by Silverman [9]. In view of Theorem 1 , since

$$
\Phi(k)=C_{k} \delta(n, k),
$$

is an increasing function of $k(k \geq 2)$, we have

$$
\Phi(2) \sum_{k=2}^{\infty}\left|a_{k}\right| \leq \sum_{k=2}^{\infty} \Phi(k)\left|a_{k}\right| \leq(B-A)(n+1),
$$

that is

$$
\sum_{k=2}^{\infty}\left|a_{k}\right| \leq \frac{(B-A)(n+1)}{\Phi(2)}=\frac{(B-A)}{2 C_{2}} .
$$

Thus we have

$$
|f(z)| \leq|z|+\sum_{k=2}^{\infty}\left|a_{k}\right||z|^{k} \leq|z|+|z|^{2} \sum_{k=2}^{\infty}\left|a_{k}\right|,
$$

Thus

$$
|f(z)| \leq|z|+\frac{(B-A)}{2 C_{2}}|z|^{2} .
$$

Similarly, we get

$$
|f(z)| \geq|z|-\sum_{k=2}^{\infty}\left|a_{k}\right||z|^{k} \geq|z|-|z|^{2} \sum_{k=2}^{\infty}\left|a_{k}\right| .
$$


Thus

$$
|f(z)| \geq|z|-\frac{(B-A)}{2 C_{2}}|z|^{2} .
$$

This completes the proof of Theorem 2. Finally the result is sharp for the function

$$
f(z)=z+\frac{(B-A)}{2 C_{2}} e^{i \theta_{2}} z^{2}
$$

at $z= \pm|z| e^{-i \theta_{2}}$.

Corollary 2. Under the hypotheses of Theorem 2, $f(z)$ is included in a disc with center at the origin and radius $r_{1}$ given by

$$
r_{1}=1+\frac{(B-A)}{2 C_{2}} \text {. }
$$

Theorem 3. Let the function $f(z)$ defined by (1.1) be in the class $V Q(n, \lambda, A, B)$. Then

$$
1-\frac{(B-A)}{C_{2}}|z| \leq\left|f^{\prime}(z)\right| \leq 1+\frac{(B-A)}{C_{2}}|z| .
$$

The result is sharp.

Proof. Similarly $\frac{\Phi(k)}{k}$ is an increasing function of $k(k \geq 2)$, where $\Phi(k)$ is defined by (3.2). In view of Theorem 1 , we have

$$
\frac{\Phi(2)}{2} \sum_{k=2}^{\infty} k\left|a_{k}\right| \leq \sum_{k=2}^{\infty} \Phi(k)\left|a_{k}\right| \leq(B-A)(n+1),
$$

that is

$$
\sum_{k=2}^{\infty} k\left|a_{k}\right| \leq \frac{(B-A)}{\Phi(2)}=\frac{(B-A)}{C_{2}}
$$

Thus we have

$$
\left|f^{\prime}(z)\right| \leq 1+|z| \sum_{k=2}^{\infty} k\left|a_{k}\right| \leq 1+\frac{(B-A)}{C_{2}}|z| .
$$

Similarly

$$
\left|f^{\prime}(z)\right| \geq 1-|z| \sum_{k=2}^{\infty} k\left|a_{k}\right| \geq 1-\frac{(B-A)}{C_{2}}|z| .
$$

Finally, we can see that the assertions of Theorem 3 are sharp for the function $f(z)$ defined by (3.4). This completes the proof of Theorem 3. 
Corollary 3. Under the hypotheses of Theorem $3, f^{\prime}(z)$ is included in a disc with center at the origin and radius $r_{2}$ given by

$$
r_{2}=1+\frac{(B-A)}{C_{2}} .
$$

\section{Extreme Points}

Theorem 4. Let the function $f(z)$ defined by (1.1) be in the class $V Q(n, \lambda, A, B)$, with $\arg a_{k}=\theta_{k}$, where $\theta_{k}+(k-1) \delta \equiv \pi(\bmod 2 \pi)(k \geq 2)$. Define

$$
f_{1}(z)=z
$$

and

$$
f_{k}(z)=z+\frac{(B-A)(n+1)}{k C_{k} \delta(n, k)} e^{i \theta_{k}} z^{k} \quad(k \geq 2 ; z \in U) .
$$

Then $f(z) \in V Q(n, \lambda, A, B)$ if and only if $f(z)$ can expressed in the form $f(z)=$ $\sum_{k=1}^{\infty} \mu_{k} f_{k}(z)$, where $\mu_{k} \geq 0$ and $\sum_{k=1}^{\infty} \mu_{k}=1$.

Proof. If $f(z)=\sum_{k=1}^{\infty} \mu_{k} f_{k}(z)$ with $\mu_{k} \geq 0$ and $\sum_{k=1}^{\infty} \mu_{k}=1$, then

$$
\begin{aligned}
& \sum_{k=2}^{\infty}\left[k C_{k} \delta(n, k)\right] \frac{(B-A)(n+1)}{k C_{k} \delta(n, k)} \mu_{k}=\sum_{k=2}^{\infty}(B-A)(n+1) \mu_{k} \\
= & \left(1-\mu_{1}\right)(B-A)(n+1) \leq(B-A)(n+1) .
\end{aligned}
$$

Hence $f(z) \in V Q(n, \lambda, A, B)$.

Conversely, let the function $f(z)$ defined by (1.1) be in the class $V Q(n, \lambda, A, B)$, define

$$
\mu_{k}=\frac{k C_{k} \delta(n, k)}{(B-A)(n+1)}\left|a_{k}\right| \quad(k \geq 2)
$$

and

$$
\mu_{1}=1-\sum_{k=2}^{\infty} \mu_{k}
$$

From Theorem $1, \sum_{k=2}^{\infty} \mu_{k} \leq 1$ and so $\mu_{1} \geq 0$. Since $\mu_{k} f_{k}(z)=\mu_{k} z+a_{k} z^{k}$, then

$$
\sum_{k=1}^{\infty} \mu_{k} f_{k}(z)=z+\sum_{k=2}^{\infty} a_{k} z^{k}=f(z) .
$$

This completes the proof of Theorem 4 . 


\section{Remarks.}

(i) Putting $\lambda=n=0, A=2 \alpha-1(0 \leq \alpha<1)$ and $B=1$ in all the above results, we obtain the corresponding results obtained by Srivastava and Owa [10];

(ii) Putting $n=0$ in all the above results, we obtain the corresponding results for the class $\operatorname{VR}(\lambda, A, B)$ of which $R(\lambda, A, B)$ is given by (1.9);

(iii) Putting $\lambda=0$ in all the above results, we obtain the corresponding results for the class $V Q_{n}(A, B)$ of which $Q_{n}(A, B)$ is given by (1.10).

\section{References}

[1] H. S. Al-Amiri, On Ruscheweyh derivatives, Ann. Polon. Math., 38(1980), 87-94.

[2] H. S. Al-Amiri, On a subclass of close-to-convex functions with negative coefficients, Math. (Cluj), 31(1989), 1-7.

[3] O. Altintas, A subclass of analytic functions with negative coefficients, Hacettepe Bull. Natur. Sci. Engrg., 19(1990), 15-24.

[4] A. A. Attiya and M. K. Aouf, A study on certain class of analytic functions defined by Ruscheweyh derivative, Soochow J. Math., 33(2)(2007), 273-289.

[5] V. P. Gupta and P. K. Jain, Certain classes of univalent functions with negative coefficients II, Bull. Austral. Math. Soc., 15(1976), 467-473.

[6] S. Owa and M. K. Aouf, On subclasses of univalent functions with negative coefficients II, Pure Appl. Math. Sci., 29(1:2)(1989), 131-139.

[7] St. Ruscheweyh, New criteria for univalent functions, Proc. Amer. Math. Soc., 49(1975), 109-115.

[8] S. M. Sarangi and B. A. Uralegaddi, The radius of convexity and starlikeness for certain classes of analytic functions with negative coefficients I, Rend. Acad. Naz. Lincei, 65(1978), 38-42.

[9] H. Silverman, Univalent functions with varying arguments, Houston J. Math., 17(1981), 283-287.

[10] H. M. Srivastava and S. Owa, Certain classes of analytic functions with varying arguments, J. Math. Anal. Appl., 136(1)(1988), 217-228.

[11] B. A. Uralegaddi and S. M. Sarangi, Some classes of univalent functions with negative coefficients, An. Stiint. Univ. Al. I. Cuza Iasi Sect. I a Mat. (N. S.), 34(1988), 7-11. 